\title{
Evaluation of the Effect of Platelet-Rich Plas- ma on Temporomandibular Joint Disorders: A Split-Match Randomized Clinical Trial
}

\section{Sezavar', Sh Shafaei Fard ${ }^{1}, H$ Sharifzadeh $^{2}, \mathbf{R}$ Pahlevan * 2, A Badkoobeh ${ }^{3}$}

1- Assistant Professor, Oral and Maxillofacial surgery Dept, Dental Faculty, Tehran Medical Sciences, Islamic Azad University, Tehran, Iran

2- Post graduate student, Oral and Maxillofacial surgery Dept, Dental Faculty, Tehran Medical Sciences, Islamic Azad University, Tehran, Iran

3- Post graduate student of oral and maxillofacial surgery, Department of Oral and Maxillofacial surgery, School of Dentistry, Shahid Beheshti University of Medical Sciences, Tehran, Iran

\begin{tabular}{l}
\hline ARTICLE INFO \\
\hline Article History \\
Received: Apr2020 \\
Accepted: May 2020 \\
ePublished:Jun 2020 \\
\hline Corresponding author: \\
R Pahlevan,Post grad- \\
uate student, Oral and \\
Maxillofacial surgery \\
Dept, Dental Faculty, \\
Tehran Medical Scienc- \\
es, Islamic Azad Univer- \\
sity, Tehran, Iran \\
Email: Drrhpn@gmail. \\
com
\end{tabular}
com

\begin{abstract}
Background and Aim: The reduction of temporomandibular joint disorders (TMD) is one of the most significant concerns in dentistry. In contrast to initial treatments, such as pharmacotherapy and physiotherapy, which act as temporary tranquilizers, growth factors have shown promising results in the stimulation of cell division. Given the limited research on the positive impact of platelet-rich plasma (PRP) on the permanent treatment of joint disorders in the knee and the temporomandibular joint (TMJ), we sought to investigate the effect of PRP on TMD.

Materials and Methods: Twenty patients (40 sides) presenting with TMD from 20112016 were included in this split-mouth clinical trial. TMD was documented by evaluating pain, limitation in movement, joint sounds, and deviation of the mandible during mouth opening. Next, $0.6 \mathrm{ml}$ of PRP prepared from subjects' blood was injected into the joints. Patients were followed-up 2, 4, and 6 months postoperatively. The severity of pain was evaluated using Mann-U-Whitney test. The other criteria were analyzed using chi-square test at the follow-up sessions and McNemar's test within each group. Result: The analysis of the results showed no difference between the two groups at the two-month follow-up. The pain intensity reduced significantly at 4- and 6-month follow-ups $(\mathrm{P}<0.05)$. Limitation in movement and joint sounds decreased from the second- to the sixth-month follow-up $(\mathrm{P}<0.05$ and $<0.02)$. The treatment seemed to have no impact on mandibular deviation $(\mathrm{P}<0.9)$.

Conclusion:Treatment with PRP seems to be a favorable adjunct in several criteria of TMD.
\end{abstract}

Keywords: Pain, Platelet-Rich Plasma, Temporomandibular Joint, Temporomandibular Disorders

\section{J Res Dent Maxillofac Sci 2020;5(3):7-14}

\section{Introduction:}

The reduction of temporomandibular joint disorders (TMD) is one of the most important issues in dentistry. TMD is one of the major oral problems with a $4 \%$ worldwide prevalence. ${ }^{(1)}$

Although the initial approach in TMD is the use of tranquilizers with temporary effects on the temporomandibular joint (TMJ) biochemical space, other approaches include noninvasive treatments such as physiotherapy, the use of a stabilization splint, and pharmacotherapy, including the prescription of acetaminophen and nonsteroidal antiinflammatory drugs (NSAIDS). ${ }^{(1-3)}$ Other minimally invasive treatments, such as lavage, hyaluronic acid and corticosteroid injections, arthrocentesis, and arthroscopy, may be used if the symptoms persist. (2)

According to different reports and research, joint space injections of hyaluronic acid and 
steroids, though not universally accepted, have shown promising results in reducing TMD signs and symptoms. ${ }^{(4)}$ Considering the lack of vascularization of the cartilage tissue and its low healing potential, various modern methods have focused on stimulation and repair of this tissue using matrix metalloproteinase, cytokine or calcitonin inhibitors, pain controllers, bisphosphonates, and growth factors. Among all the aforementioned substances, growth factors are preferable as they stimulate cell division and joint repair. ${ }^{(1,5)}$

There is a lack of sufficient data regarding the application of platelet-rich plasma (PRP) in TMD treatment while promising reports have been published on the use of this substance in the knee. Therefore, we sought to investigate the efficacy of PRP in reducing TMD at the Oral and Maxillofacial Surgery Department, Faculty of Dentistry, Islamic Azad University of Medical Sciences and Bu Ali Hospital, Tehran, Iran, during 2011-2016.

\section{Materials and Methods:}

In this split-match randomized clinical trial, all patients diagnosed with TMD, who referred to the Oral and Maxillofacial Surgery Department of the Faculty of Dentistry of Islamic Azad University of Medical Sciences and Bu Ali Hospital, Tehran, Iran, (2011-2016) were investigated. After project justification, they agreed to cooperate and present at the centers mentioned above at the given time. Patients signed informed consent forms before the study. Subjects who met all the following requirements were included in the study: a clinical history of over 6 months of TMD that alleviates with mandibular movement in function or parafunction, pain in clinical examination during mouth opening, laterality or palpation, and no previous treatment. All patients were eligible candidates for PRP injection. The Ethics Committee of Islamic Azad University of Medical Sciences approved the protocol of this study (IRCT20180503039510N1).

Cases with specific conditions such as systemic health problems and diseases, rheumatic pathologies such as rheumatoid arthritis or psoriatic arthritis (including juvenile arthritis), TMJ surgery candidates, pregnant or breastfeeding women, patients with a non-reducing disc dis- placement, and those who were under 18 years of age were excluded from the study. Selected cases were prescribed with acetaminophen codeine $(522 \mathrm{mg})$ for two weeks before PRP injection.

PRP Preparation: First, 6cc of blood was drawn by phlebotomy. The blood was transferred from the syringe to sterile tubes coated with an anticoagulant substance $(2.3 \%$ dextrose citrate and sodium citrate) and was centrifuged for 3 minutes at 1300 revolutions per minute (rpm). Subsequently, three layers were formed in the tube, including red blood cells, a buffy coat (a combination of platelets and white blood cells), and acellular plasma, from the deepest layer to the top, respectively. The acellular plasma layer was removed with great caution. The buffy coat (PRP) was collected using a pipette, and eventually, PRP was injected into the joint.

The injection site was disinfected using povidone-iodine (Betadine). The site of injection was $10 \mathrm{~mm}$ anterior to the tragus-canthus line and 2 $\mathrm{mm}$ below this point. Located $20 \mathrm{~mm}$ from the tragus-canthus line and $7 \mathrm{~mm}$ below the tragus was the second injection site. ${ }^{(1)}$

TMDs were classified in 1992 as Research Diagnostic Criteria (RDC/TMD), which are broadly divided into three groups: muscle disorders (group I), disc displacement disorders (group II), and other TMJ disorders, such as osteoarthritis and arthrosis (group III). ${ }^{(6)}$ The following parameters were analyzed in detail: changes in the intensity of pain as reported by patients concerning the TMJ and the stomatognathic system muscles, pain intensity characteristics, level of impairment, and grade of chronic pain. ${ }^{(6)}$ The assessment also involved a subjective evaluation of pain within the stomatognathic system muscles and the TMJ, evaluation of the range and symmetry of the mandible's motion, acoustic symptoms within the TMJ, and the impact of the masticatory organ health on overall well-being. ${ }^{(6,7)}$

Two $20 \mathrm{G}$ needles were utilized. Thereafter, $2 \mathrm{ml}$ of saline solution was injected to ensure proper marking of the joint space. Then, $0.6 \mathrm{ml}$ of PRP was gently injected into the superior joint space. A soft diet was prescribed for one week. Anti-inflammatory drugs were prohibited to avoid the analgesic effects and alteration of the study results regarding pain relief.

After intra-articular (superior space) injection of 
PRP, the study variables consisting of pain, mandibular deviation during mouth opening, joint sounds, and limitation in jaw movements during mouth opening were investigated immediately and 2, 4, and 6 months postoperatively and were recorded in a form. Patients' pain was statistically analyzed using Mann-U-Whitney test at each follow-up session and Wilcoxon test within each group. The indicators (limitation in jaw movement, joint sounds, and mandibular deviation) were statistically analyzed via chi-square test at the follow-up sessions and using McNemar's test within each group.

\section{Results:}

The present study involved 20 patients representing 40 samples ( $40 \%$ male and $60 \%$ female) with an average age of $38 \pm 13.2$ years. The samples were matched in both groups according to the split-match study protocol.
The pain intensity remained unchanged immediately after treatment and two months postoperatively. This intensity was $3.7 \pm 1.38$ and $2.5 \pm 1.87$ at the fourth-month follow-up for the control and experimental groups, respectively. Furthermore, at the 6th-month follow-up, the pain intensity was $3.3 \pm 1.41$ and $2.2 \pm 1.76$ for the control and experimental groups, respectively $(\mathrm{P}<0.05)$.

Regarding limitations in the jaw movement, the measures in the two groups were equal, and there was a significant gradual reduction in the experimental group from the second- to the sixth-month follow-up $(\mathrm{P}<0.05)$. Joint sounds were evaluated at the second follow-up session and were diagnosed in $75 \%$ of the patients in the control group and $40 \%$ in the experimental group $(\mathrm{P}<0.02)$. Such results were also observed during the follow-ups. No significant difference was detected in mandibular deviation after PRP injection $(\mathrm{P}<0.9)$.

Table 1: Distribution of patients with temporomandibular disorders (TMD) by pain intensity after platelet-rich plasma (PRP) injection and during the follow-up sessions

\begin{tabular}{lllll} 
& $\begin{array}{l}\text { Pain } \\
\text { Preoperative } \\
\text { (Zero) }\end{array}$ & $2^{\text {nd }}$ month & $4^{\text {th }}$ month & $6^{\text {th }}$ month \\
\hline $\mathrm{NO}$ & $1.27 \pm 4.95$ & $1.38 \pm 4.1$ & $1.38 \pm 3.7$ & $1.41 \pm 3.3$ \\
$($ Control $)$ & $1.7 \pm 4.5$ & $1.95 \pm 3.7$ & $1.87 \pm 2.5$ & $1.76 \pm 2.2$ \\
\hline Yes & $\mathrm{P}<0.4$ & $\mathrm{P}<0.6$ & $\mathrm{P}<0.05$ & $\mathrm{P}<0.05$ \\
\hline
\end{tabular}

Table 2: Distribution of patients with temporomandibular disorders (TMD) by limitation in movement after platelet-rich plasma (PRP) injection and during the follow-up sessions

\begin{tabular}{lllllllll}
\hline & $\begin{array}{c}\text { Limitation in } \\
\text { movement }\end{array}$ & $\begin{array}{l}\text { Preoperative } \\
\text { (Zero) }\end{array}$ & & 2nd month & 4th month & \multicolumn{3}{c}{ 6th month } \\
\cline { 2 - 9 } PRP & No & Yes & No & Yes & No & Yes & No & Yes \\
\hline $\begin{array}{l}\text { NO } \\
\text { (Control) }\end{array}$ & $14(70)$ & $7(30)$ & 16 & 4 & $18(90)$ & $2(10)$ & $18(90)$ & $2(10)$ \\
\hline $\begin{array}{l}\text { Yes } \\
\text { (Experimental) }\end{array}$ & $14(70)$ & $6(20)$ & 16 & 4 & $18(90)$ & $2(10)$ & $19(95)$ & $8(5)$ \\
\hline Result & $\mathrm{P}<0.9$ & & $\mathrm{P}<0.9$ & & $\mathrm{P}<0.9$ & & $\mathrm{P}<0.3$ & \\
\hline
\end{tabular}


Table 3: Distribution of patients with temporomandibular disorders (TMD) by joint sounds after platelet-rich plasma (PRP) injection and during the follow-up sessions

\begin{tabular}{|c|c|c|c|c|c|c|c|c|}
\hline \multirow{2}{*}{ PRP } & \multicolumn{2}{|c|}{$\begin{array}{l}\text { Preoperative } \\
\text { (Zero) }\end{array}$} & \multicolumn{2}{|c|}{$2^{\text {nd }}$ month } & \multicolumn{2}{|c|}{$4^{\text {th }}$ month } & \multicolumn{2}{|c|}{$6^{\text {th }}$ month } \\
\hline & No & Yes & No & Yes & No & Yes & No & Yes \\
\hline $\begin{array}{l}\text { NO } \\
\text { (Control) }\end{array}$ & $5(20)$ & $15(60)$ & $5(20)$ & $15(75)$ & $6(30)$ & $14(70)$ & $9(45)$ & $11(55)$ \\
\hline $\begin{array}{l}\text { Yes } \\
\text { (Experimental) }\end{array}$ & $7(35)$ & 13 & $12(60)$ & $8(40)$ & $14(70)$ & $6(30)$ & $5(75)$ & $5(52)$ \\
\hline Result & $\mathrm{P}<0.5$ & & $\mathrm{P}<0.025$ & & $\mathrm{P}<0.02$ & & $\mathrm{P}<0.06$ & \\
\hline
\end{tabular}

The distribution of the subjects by limitation in movement during the follow-up sessions is shown in Table 2 representing that:

- Limitation in movement in both groups was equal before and during the follow-up sessions, and the differences were not statistically significant, suggesting that PRP injection did not affect limitation in movement $(\mathrm{P}<0.3)$.

- During the intra-group investigations, we found no subjects in the control group suffering from limitation in movement but a significant reduction was found from $6(20 \%)$ to $3(10 \%)$ at the 4th and 6th follow-up sessions, respectively. McNemar's test showed that this effect was statistically significant from the fourth month onwards $(\mathrm{P}<0.5)$. Within the experimental group, no effect was found at the second follow-up session (second month) but a significant reduction was found at the fourth (from $20 \%$ to $10 \%$; $<<0.05$ ) and 6th-month follow-ups (from $20 \%$ to $5 \%$ ). This effect was statistically significant $(\mathrm{P}<0.05)$. The distribution of the subjects by joint sounds after PRP injection is presented in Table 3, suggesting that:

- At the beginning of the study (time zero), joint sounds were reported in 15 subjects of the con- trol group and 13 subjects of the experimental group. The test showed that both groups were similar, and the differences were not statistically significant $(\mathrm{P}<0.5)$.

- At the second-month follow-up session, joint sounds were still reported for 15 and 8 subjects in the control and experimental groups, respectively. The test showed that this difference $(35 \%$; 40-75) was statistically significant $(\mathrm{P}<0.02)$.

- At the 4th-month follow-up, joint sounds were reported for 14 subjects in the control group and 6 subjects in the experimental group (40\% lower than the control group), which is statistically significant $(\mathrm{P}<0.02)$.

- After six months, joint sounds were reported for 11 subjects in the control group and 5 subjects in the experimental group $(30 \%$ lower than the control group; $\mathrm{P}<0.06$ ).

- No significant difference was observed in joint sounds in the control group after six months $(\mathrm{P}<0.02)$. In the experimental group at the second follow-up session, the study showed a decrease in joint sounds followed by a rise from $35 \%$ to $60 \%$. Joint sounds showed a significant reduction of $70 \%$ at the 4 th-month follow-up $(\mathrm{P}<0.03)$. 
At the beginning of the study (time zero), mandibular deviation during mouth opening was reported for three subjects in the control group (15\%) similar to the experimental group $(\mathrm{P}<0.9)$. Throughout the follow-up sessions, no changes were experienced in this regard.

\section{Discussion:}

The current study shows that PRP injection into the TMJ for treatment of patients with TMD is an=effective mean to positively affect some indicators such as pain, as well as to reduce joint sounds and limitation in movement; however, this treatment did not affect mandibular deviation during mouth opening. Pihut et al sought to investigate pain relief in patients with TMJ dysfunction. ${ }^{(1)}$

They performed PRP injection for 10 cases (male and female) with the average age of 28-53 years. The results were measured 7 days and 6 weeks later using a Visual Analog Scale (VAS). They reported the positive effect of PRP in reducing the severity of pain in the TMJ.(1) In the study by Pihut et al, no control group was considered, and among all TMJ dysfunctions, only the pain was investigated, while in TMD, it is not merely pain that must be treated. Besides, the time intervals to survey the results were shorter than that in the current research.

Hanci et al compared the benefit of PRP injection with that of arthrocentesis and showed a statistically significant reduction in pain intensity and joint sounds as well as an increase in mouth opening in the study group compared to the control group. ${ }^{(4)}$ The results of the present study are similar to that of Hanci et al. Although beneficial, the study by Hanci et al comprised shortcomings in that the reason to compare PRP injection with arthrocentesis was not mentioned; moreover, the groups were not matched according to the splitmatch study protocol while in the present study, we used the mentioned method plus non-parametric statistical tests. Above all, the research by Hanci et al did not consider the changes within the groups. ${ }^{(4)}$

In a systematic review conducted by Bousnaki et al, the effect of PRP therapy on TMD was examined. ${ }^{(2)}$ After reviewing the articles published until May 2017, six papers were selected from among 153 articles and were matched according to the inclusion criteria presented by the researchers. ${ }^{(2)}$ It was concluded that there is little benefit in using PRP for TMD treatments. However, the results deemed a PRP standard preparation protocol necessary. A prominent cause of the difference between the current study and the study by Bousnaki et al was the inclusion of cases of osteoarthritis in their paper while patients suffering from systemic diseases were excluded from our study. Consequently, the difference between the two results seems to be due to the minor efficiency of PRP injection in patients with osteoarthritis compared to those without special diseases.

Simsek studied seven females with TMD and injected PRP twice on both sides. ${ }^{(8)}$ After three months (the last injection), pain intensity was assessed using a VAS. The study showed the reduction of the initial index from $7.66 \pm 1.3$ to $5.33 \pm 2$ after the first injection and to $3.33 \pm 1.2$ after the second injection. Reduction in pain intensity was reported after the first injection while changes after the second injection were more significant. (8) No control group was considered in the mentioned study. In addition, all patients were females, which can decrease the value and merit of the study. In the research by Simsek, among all TMJ dysfunctions, only pain intensity was the focus of study, while in our research, not only pain but also other indicators were considered, and only $60 \%$ of the subjects were females. Eventually, both studies found a positive PRP effect on pain relief.

Considered as an organic treatment modality, PRP encompasses platelets concentrated from a given patient's blood. ${ }^{(9)}$ This enriched plasma, which can even increase up to $2,000,000$ units/ $\mathrm{ml}$, is the result of blood centrifugation aiming at obtaining significant amounts of platelets. ${ }^{(10)}$ Specific proteins, such as fibrin, vitronectin, and fibronectin, which can help cell adhesion, are found in PRP. Hence, PRP can stimulate regeneration in body tissues through the stimulation of fibroblasts to produce some fundamental proteins, which are used in the formation of elastin and collagen, remodeling and formation of new vessels (angiogenesis), and activation of stem cells renowned as mesenchymal cells. ${ }^{(1)}$ 
Accordingly, pain or dysfunction, which can be witnessed when the mandible moves, could be pertained to the pressure that increases within the joints, as well as the high amount of secreted cytokines (interleukin, interferon, and growth factors) available in the synovial liquid. ${ }^{(1)}$ Not only does PRP improve the regeneration of cartilage but also it can repair hard tissues. ${ }^{(12)}$ It could be deciphered that, in the present study, injection of PRP takes significance because this autogenous agent has been able to upsurge osteoblastic activity, leading to dense mature bone formation in patients. ${ }^{(3,12)}$

Furthermore, PRP can stimulate cell proliferation and cartilage matrix production through chondrocytes and mesenchymal stromal cells, which are bone marrow-derived, and has the capacity of enhancing hyaluronic acid production by the help of synoviocytes. ${ }^{(13)}$ PRP can modulate the biology of synovial cells that play a significant role in restoring discs, capsules, and retrodiscal pads. Likewise, it has the capacity of inhibiting pro-inflammatory cytokines. ${ }^{(12,14)}$

It can be argued that PRP enhances tissue repair while subsiding tissue breakdown, and it has antibacterial, analgesic, and anti-inflammatory properties. Likewise, this enriched plasma offers some regenerative properties, which can increase the concentration of hyaluronic acid in combination with an increase in the chondrocyte synthesis of glycosaminoglycan. PRP can also balance joint angiogenesis as it develops a scaffold to be used for stem cell migration. ${ }^{(15,16)}$ Additionally, various growth factors are embedded in PRP. ${ }^{(3,12)}$ Such factors, which guarantee its efficiency and significant impact, are likely to affect TMD treatment more efficiently in comparison with hyaluronic acid injection or arthrocentesis. ${ }^{(17)}$

Moreover, PRP-supported treatments can inhibit pro-inflammatory cytokines. In case this inhibition does not take place, pro-inflammatory cytokines may impede tissue healing through interleukin (IL)-1 release suppression. ${ }^{(14)}$ Besides, PRP can inhibit IL-1b, tumor necrosis factoralpha, and nuclear factor-kappa B (NF-kB) catabolic effects on the cartilage. ${ }^{(18)}$ The aforementioned notions signify that PRP has a dual action: a) inhibiting tissue degradation, and b) enhancing healing properties of the cells. When PRP is injected intra-articularly, it could sustain the chon- dral surface integrity as it accelerates joint movement. ${ }^{(19)}$

Despite its limitedly known action mechanism, PRP has gained popularity in orthopedics and sports medicine and has been effectively used in the treatment of osteoarthritis and cartilage defects.

Lately, PRP has been injected intra-articularly into the TMJ for patients afflicted by disc displacement and TMJ osteoarthritis. Though the literature has extensively accounted for various applications of PRP in joints like the knee, employing this substance in the treatment of TMJ has recorded a step forward in terms of novelty. Several techniques have been adopted to isolate PRP with different contents. Based on the concentration of its platelets, leucocytes, and fibrin, PRP enjoys unique biology and specific applications. Currently, with regard to their cell content and fibrin, four large families of platelet concentrates are taken into consideration: a) leukocyteand platelet-rich plasma (LPRP, b) leukocyte- and platelet-rich fibrin (L-PRF), c) pure platelet-rich plasma (P-PRP), and d) pure platelet-rich fibrin (P-PRF). The focus of the present study was on the application of pure PRP in the treatment of TMD. ${ }^{(20,21)}$

A remarkable aspect of this study is the use of a split-match method. Since no financial support was utilized, there is no orientation in favor of PRP. Another significant feature is various intra and extra group statistical tests that increase the accuracy of the research.

Due to a lack of TMD cases at the Faculty of Dentistry, most of the cases were referred from $\mathrm{Bu}$ Ali Hospital; this would reduce the external validity of this study. Although the patients signed informed consent forms and all were justified about the project, there were various problems with their follow-up sessions.

In previous studies, as well as the present study, reduction of joint sounds after PRP injection has been reported, which can be due to a reduction in articular inflammatory processes. ${ }^{(1,2,12)}$ This symptom can be suggested as a guideline to examine the status of the disc using magnetic resonance imaging (MRI) before and after PRP treatment. 


\section{Conclusion:}

According to the findings, PRP injection seems to have a positive effect on pain, limitation in jaw movement, and joint sounds. This effect is significant from the fourth month of injection.

\section{Acknowledgements:}

We would like to extend our gratitude to Mr. Naser Valaei, the consultant of the statistics and methodology of the study. We also wish to thank all the participants of the project at the Oral and Maxillofacial Surgery Department, Faculty of Dentistry, Islamic Azad University of Medical Sciences and Bu Ali Hospital, Tehran, Iran.

\section{References:}

1. Pihut M, Szuta M, Ferendiuk E, ZeńczakWięckiewicz D. Evaluation of pain regression in patients with temporomandibular dysfunction treated by intra-articular platelet-rich plasma injections: a preliminary report. Biomed Res Int. 2014;2014:132369.

2. Bousnaki M, Bakopoulou A, Koidis P. Platelet-rich plasma for the therapeutic management of temporomandibular joint disorders: a systematic review. Int J Oral Maxillofac Surg. 2018 Feb;47(2):188-98.

3. Kurita Varoli F, Sucena Pita M, Sato S, Issa JP, do Nascimento C, Pedrazzi V. Analgesia evaluation of 2 NSAID drugs as adjuvant in management of chronic temporomandibular disorders. ScientificWorldJournal. 2015;2015:359152.

4. Hanc1 M, Karamese M, Tosun Z, Aktan TM, Duman S, Savaci N. Intra-articular platelet-rich plasma injection for the treatment of temporomandibular disorders and a comparison with arthrocentesis. J Craniomaxillofac Surg. 2015 Jan;43(1):162-6.

5. Cömert Kiliç $S$, Güngörmüş $M$, Sümbüllü MA. Is Arthrocentesis Plus Platelet-Rich Plasma Superior to Arthrocentesis Alone in the Treatment of Temporomandibular Joint Osteoarthritis? A Randomized Clinical Trial. J Oral Maxillofac Surg. 2015 Aug;73(8):1473-83.

6. Schiffman E, Ohrbach R, Truelove E, Look $\mathrm{J}$, Anderson G, Goulet JP, et al. International RDC/TMD Consortium Network, International association for Dental Research; Orofacial Pain Special Interest Group, International Associa- tion for the Study of Pain. Diagnostic Criteria for Temporomandibular Disorders (DC/TMD) for Clinical and Research Applications: recommendations of the International RDC/TMD Consortium Network and Orofacial Pain Special Interest Group $\dagger$. J Oral Facial Pain Headache. 2014 Winter;28(1):6-27.

7. Dworkin SF, LeResche L. Research diagnostic criteria for temporomandibular disorders: review, criteria, examinations and specifications, critique. J Craniomandib Disord. 1992;6(4):301355.

8. Simsek ME. Bilateral platelet rich plasma injections with assisted techniques for temporomandibular joint disorders. Eur Res J. 2016 2:2149-3189.

9. Pietrzak WS, Eppley BL. Platelet rich plasma: biology and new technology. J Craniofac Surg. 2005 Nov;16(6):1043-54.

10.Eppley BL, Woodell JE, Higgins J. Platelet quantification and growth factor analysis from platelet-rich plasma: implications for wound healing. Plast Reconstr Surg. 2004 Nov;114(6):15028.

11.Fernández-Ferro $\quad M$, Fernández-Sanromán J, Blanco-Carrión A, Costas-López A, LópezBetancourt A, Arenaz-Bua J, et al. Comparison of intra-articular injection of plasma rich in growth factors versus hyaluronic acid following arthroscopy in the treatment of temporomandibular dysfunction: A randomised prospective study. J Craniomaxillofac Surg. 2017 Apr;45(4):449-54.

12.Kütük N, Baş B, Soylu E, Gönen ZB, Yilmaz $\mathrm{C}$, Balcioğlu E, et al. Effect of platelet-rich plasma on fibrocartilage, cartilage, and bone repair in temporomandibular joint. J Oral Maxillofac Surg. 2014 Feb;72(2):277-84.

13.Hegab AF, Ali HE, Elmasry M, Khallaf MG. Platelet-Rich Plasma Injection as an Effective Treatment for Temporomandibular Joint Osteoarthritis. J Oral Maxillofac Surg. 2015 Sep;73(9):1706-13.

14. Woodall J Jr, Tucci M, Mishra A, Asfour A, Benghuzzi $\mathrm{H}$. Cellular effects of platelet rich plasmainterleukin1 release from prp treated macrophages. Biomed Sci Instrum. 2008;44:489-94. 15.Civinini R, Macera A, Nistri L, Redl B, Innocenti $\mathrm{M}$. The use of autologous blood-derived growth factors in bone regeneration. Clin Cases Miner Bone Metab. 2011 Jan-Apr;8(1):25-31. 
16.Anitua E, Sánchez M, Nurden AT, Zalduendo MM, de la Fuente M, Azofra J, et al. Plateletreleased growth factors enhance the secretion of hyaluronic acid and induce hepatocyte growth factor production by synovial fibroblasts from arthritic patients. Rheumatology (Oxford). 2007 Dec;46(12):1769-72.

17.Zotti F, Albanese M, Rodella LF, Nocini PF. Platelet-Rich Plasma in Treatment of Temporomandibular Joint Dysfunctions: Narrative Review. Int J Mol Sci. 2019 Jan 11;20(2):277.

18.van Buul GM, Koevoet WL, Kops N, Bos PK, Verhaar JA, Weinans $\mathrm{H}$, et al. Platelet-rich plasma releasate inhibits inflammatory processes in osteoarthritic chondrocytes. Am J Sports Med. 2011 Nov;39(11):2362-70.

19.Lippross S, Moeller B, Haas H, Tohidnezhad $\mathrm{M}$, Steubesand N, Wruck CJ, et al. Intraarticular injection of platelet-rich plasma reduces inflammation in a pig model of rheumatoid arthritis of the knee joint. Arthritis Rheum. 2011 Nov;63(11):3344-53.

20.Dohan Ehrenfest DM, Rasmusson L, Albrektsson T. Classification of platelet concentrates: from pure platelet-rich plasma (P-PRP) to leucocyte- and platelet-rich fibrin (L-PRF). Trends Biotechnol. 2009 Mar;27(3):158-67.

21. Azizi A, Dabirmoghadam P, Keykha F. Comparison of the Concentration of Salivary IL-8 in Patients with Oral Squamous Cell Carcinoma and Healthy Subjects . J Res Dentomaxillofac Sci. 2016; 1 (3) :28-32.

Please cite this paper as: Sezavar M, Shafaei Fard S, Sharifzadeh H, Pahlevan R, Badkoobeh A. Evaluation of the Effect of Platelet-Rich Plasma on Temporomandibular Joint Disorders: A Split-Match Randomized Clinical Trial. J Res Dentomaxillofac Sci. 2020;5(3):7-14 